\title{
Posicionamento Estratégico e Desempenho de Mercado da Indústria Farmacêutica à Luz da Tipologia de Porter
}

\author{
Antonio Felipe de Almeida Pinho \\ Jorge Ferreira da Silva
}

\section{RESUMO}

Este estudo tem como objetivo principal identificar de que modo, na indústria farmacêutica brasileira, as estratégias escolhidas pelas empresas, durante o período compreendido entre 1995 e 1998, influenciaram os seus posicionamentos e, conseqüentemente, impactaram os seus desempenhos de mercado. Apresenta, ainda, à luz da teoria de Porter $(1980,1985)$ sobre estratégias competitivas, as estratégias relevantes para as empresas pertencentes à indústria farmacêutica brasileira durante o período pesquisado, bem como identifica os grupos estratégicos formados pelos componentes desse segmento industrial. Para tanto, foram utilizadas informações a respeito de 50 laboratórios, formando, assim, um banco de dados. Essa base de dados contém informações sobre o comportamento estratégico das empresas a partir de onze variáveis de estratégias perseguidas e de quatro variáveis de desempenho levantadas na literatura especializada. Os dados foram analisados estatisticamente com o uso destes procedimentos: análise de fatores, análise de cluster e análise multivariada da variância. Os resultados obtidos nessa análise sugerem que as diferenças de desempenho médio dos grupos estratégicos formados podem ser explicadas em função das diferenças entre as decisões estratégicas de cada empresa da amostra.

Palavras-chaves: planejamento estratégico; indústria farmacêutica; Porter; estratégia; marketing estratégico.

\begin{abstract}
The main objective of this study is to identify, in the Brazilian pharmaceutical industry, the impact of the companies strategies in their performances, during the period from1995 to 1998. Based on Porter's $(1980,1985)$ framework of competitive strategy, the research presents the significant strategies for the Brazilian pharmaceutical industry, furthermore identifies the strategic groups formed by the components that make up this industrial segment. For the data analysis were used information about 50 laboratories. This data base contains information about the strategic behaviour of the companies divided in eleven strategic variables, as well as information of their performances in four performance variables. The data were statistically evaluated using the following procedures: Factor Analysis, Cluster Analysis and Multivariate Analysis of Variance. The obtained results suggest that the differences between the average performance of the formed strategic groups can be explained by the strategic decisions of each firm considered in the sampling.
\end{abstract}

Key words: strategical planning; pharmaceutical industry; Porter; strategy; strategical marketing. 


\section{INTRODUÇÃO}

O presente trabalho baseia-se no paradigma estratégia-desempenho para investigar a indústria farmacêutica brasileira. O estudo analisa, à luz da tipologia proposta por Porter $(1980,1985)$, as estratégias adotadas por cada empresa da indústria, verificando os seus efeitos quanto ao posicionamento e o desempenho por elas obtidos. Neste sentido, o estudo tem como objetivo final identificar, na indústria farmacêutica brasileira, de que maneira as estratégias escolhidas pelas empresas influenciam os seus posicionamentos e, conseqüentemente, impactam os seus desempenhos. Além do objetivo final, a pesquisa possui ainda quatro objetivos intermediários: (1) identificar as estratégias competitivas relevantes para a indústria farmacêutica brasileira; (2) determinar que decisões estratégicas se fariam de maior importância para a identificação de grupos estratégicos nessa mesma indústria; (3) analisar as empresas da indústria farmacêutica brasileira de acordo com as decisões estratégicas determinadas no item 2, de modo que as empresas com decisões estratégicas semelhantes façam parte de um mesmo grupo estratégico; e além disso, estabelecer condições de contorno que vinculem tais decisões estratégicas e conseqüentemente os grupos estratégicos à tipologia de estratégias genéricas de liderança de custo, diferenciação, enfoque em custo e enfoque em diferenciação, proposta por Porter (1980, 1985); (4) mensurar o desempenho dos diversos grupos estratégicos objetivando avaliar as relações entre as decisões estratégicas e o desempenho das empresas, inferindo, assim, se a pertinência a determinado grupo estratégico tem implicações de desempenho para a firma.

\section{Metodologia}

\section{Seleçăo dos Sujeitos}

Na pesquisa, foram consideradas as empresas do setor farmacêutico que comercializam produtos no mercado. Nesse sentido, a amostra foi formada pelas 50 empresas que possuíam o maior faturamento em dólar no ano de 1998, segundo a base de dados do Pharmaceutical Market Brazil (PMB, 1999).

De acordo com os dados de 1999 do PMB, o mercado total conta com cerca de 280 indústrias, o que lhe confere uma característica de mercado extremamente 
fragmentado. No entanto a amostra considerada na pesquisa, com as 50 maiores empresas, abrange $92 \%$ do mercado total em termos de faturamento e $90 \% \mathrm{em}$ termos de unidades vendidas. Dessa forma, vemos que acrescentar um número maior de empresas iria aumentar muito a complexidade da análise, sem que isso aumentasse significativamente o poder explicativo do modelo.

\section{Coleta de Dados}

Seguindo a ótica de Cool e Schendel (1987) de que a definição de variáveis estratégicas depende em grande grau da indústria, a pesquisa dividiu-se em duas grandes etapas.

Na primeira etapa, foi realizada uma pesquisa bibliográfica com o objetivo de selecionar um conjunto de variáveis estratégicas que, ao mesmo tempo, possibilitasse a identificação de grupos estratégicos para a indústria farmacêutica brasileira e que permitisse o estabelecimento de condições de contorno que vinculassem as estratégias competitivas escolhidas à tipologia de estratégias genéricas proposta por Porter $(1980,1985)$.

Tomando como base as variáveis estratégicas e de desempenho adotadas por Cool (1985), que já foram utilizadas em alguns outros estudos (Cool e Schendel, 1987; Cool e Dierickx, 1993; Bogner, Thomas e McGee, 1996), foram escolhidas as variáveis presentes neste estudo; todavia, em função da não disponibilidade de informação sobre algumas variáveis, não foram consideradas todas aquelas sugeridas por Cool (1985).

Para as duas dimensões estratégicas abordadas no estudo foram utilizados indicadores objetivos, isto é, dados que fornecem informações sobre as estratégias e os desempenhos realizados e não sobre os almejados.

A segunda etapa teve início com a necessidade de coletar os dados para a realização da pesquisa. As fontes de dados utilizadas foram as consultorias específicas da indústria farmacêutica, entre as quais podemos destacar: (1) Pharmaceutical Market Brazil Plus (PMB-Plus); (2) Índice Nacional de Terapêutica e Enfermidades (INTE); (3) Close-Up; (4) Perfil Comercial dos Produtos Farmacêuticos; e (5) Promoção e Publicidade Médica (PPM). 


\section{Seleção das Variáveis}

\section{Quadro 1: Variáveis Estratégicas}

\begin{tabular}{|c|c|c|}
\hline $\mathrm{N}^{0}$ & VARIÁVEIS & DESCRIÇÃO \\
\hline 1 & $\mathrm{FOCO}$ & $\begin{array}{l}\text { Relação entre as vendas das três maiores classes terapêuticas de } \\
\text { cada laboratório e as vendas totais do laboratóno. }\end{array}$ \\
\hline 2 & RECEITUARIO & $\begin{array}{l}\text { Relação entre as vendas dos medicamentos sob prescrição }(\mathrm{Rx}) \\
\text { de cada laboratório e as vendas totais do laboratório. }\end{array}$ \\
\hline 3 & $\begin{array}{l}\text { GENÉRICO DE } \\
\text { MARCA }\end{array}$ & $\begin{array}{l}\text { Relação entre as vendas dos medicamentos chamados genéricos } \\
\text { de marca de cada laboratório e as vendas totais do laboratório. }\end{array}$ \\
\hline 4 & $\begin{array}{l}\text { GENÉRICO } \\
\text { COMMODITY }\end{array}$ & $\begin{array}{l}\text { Relação entre as vendas dos medicamentos chamados genéricos } \\
\text { commodities de cada laboratório e as vendas totais do laboratório. }\end{array}$ \\
\hline 5 & $\begin{array}{l}\text { USO } \\
\text { CRÔNICO }\end{array}$ & $\begin{array}{l}\text { Relação entre as vendas dos medicamentos de uso crônico de cada } \\
\text { laboratório e as vendas totais do laboratório. }\end{array}$ \\
\hline 6 & $\begin{array}{l}\text { PROCURA } \\
\text { ESPONTANEA }\end{array}$ & $\begin{array}{l}\text { Relação entre as vendas dos medicamentos de procura espontânea } \\
\text { de cada laboratório e as vendas totais do laboratório. }\end{array}$ \\
\hline 7 & $\begin{array}{l}\text { NOVOS } \\
\text { PRODUTOS }\end{array}$ & $\begin{array}{l}\text { Relação entre as vendas dos novos produtos (lançados nos últimos } \\
12 \text { meses) de cada laboratório e as vendas totais do laboratónio. }\end{array}$ \\
\hline 8 & $\begin{array}{l}\text { NOVAS } \\
\text { MOLECULAS }\end{array}$ & $\begin{array}{l}\text { Relação entre as vendas dos novos produtos feitos a partir de } \\
\text { novas moléculas de cada laboratório e as vendas totais do } \\
\text { laboratório. }\end{array}$ \\
\hline 9 & PROMOÇÃO & $\begin{array}{l}\text { Relação entre o total de gastos com promoção de cada laboratório } \\
\text { e as vendas totais do laboratório. }\end{array}$ \\
\hline 10 & $\begin{array}{l}\text { PRECO } \\
\text { MÉDIO }\end{array}$ & $\begin{array}{l}\text { Relação entre o valor das vendas de cada laboratório e a } \\
\text { quantidade vendida do laboratório. }\end{array}$ \\
\hline 11 & TAMANHO & $\begin{array}{l}\text { Determinado a partir do logarítmo neperiano das vendas totais de } \\
\text { cada laboratório. }\end{array}$ \\
\hline
\end{tabular}

\section{Quadro 2: Variáveis de Desempenho}

\begin{tabular}{|c|l|l|}
\hline No & \multicolumn{1}{|c|}{ VARIÁVEIS } & \multicolumn{1}{c|}{ DESCRIÇÃO } \\
\hline 1 & MARKET SHARE & $\begin{array}{l}\text { Participação das vendas dos medicamentos de cada empresa no mercado } \\
\text { doméstico total }\end{array}$ \\
\hline 2 & $\begin{array}{l}\text { MARKET SHARE PONDERADO } \\
\text { POR SEGMENTO (WWS) }\end{array}$ & $\begin{array}{l}\text { Market Share ponderado pela participação de vendas em cada classe } \\
\text { terapêtica. }\end{array}$ \\
\hline 3 & $\begin{array}{l}\text { CRESCIMENTO DO MARKET } \\
\text { SHARE }\end{array}$ & $\begin{array}{l}\text { O crescimento do Market Share é uma variável criada com base na } \\
\text { variável Market Share. Ela representa o desempenho da empresa na } \\
\text { tentativa de conquistar maiores parcelas de mercado. }\end{array}$ \\
\hline 4 & $\begin{array}{l}\text { CRESCIMENTO DO MARKET } \\
\text { SEGM PONDERADO POR }\end{array}$ & $\begin{array}{l}\text { Assim como a varúvel Crescimento do Market Share, o Crescimento do } \\
\text { Market Share ponderado foi uma variável criada com base em outra } \\
\text { variável Ela também representa a evolução da conquista de mercado, no } \\
\text { entanto, por ser ponderada pelo segmento. }\end{array}$ \\
\hline
\end{tabular}


O market share ponderado foi construído por meio da seguinte relação:

$$
\mathrm{WWS}_{\mathrm{i}}=\sum\left(\mathrm{W}_{\mathrm{j}} \mathrm{SS}_{\mathrm{j}}\right)
$$

onde:

$$
\mathrm{W}_{\mathrm{j}}=\mathrm{s}_{\mathrm{j}} \backslash \mathrm{S}_{\mathrm{i}} \quad \mathrm{SS}_{\mathrm{j}}=\mathrm{s}_{\mathrm{j}} \backslash \mathrm{S}_{\mathrm{j}}
$$

$\mathrm{s}_{\mathrm{j}}=$ Vendas da droga i no segmento (classe terapêutica) $\mathrm{j}$.

$\mathrm{S}_{\mathrm{i}}=$ Total de vendas no mercado ético da firma $\mathrm{i}$.

$\mathrm{S}_{\mathrm{j}}=$ Volume total do mercado do segmento (classe terapêutica) $\mathrm{j}$.

O market share ponderado por segmento mede a dominação pela firma do segmento de mercado no qual compete. Esse indicador foi descrito como altamente relacionado com lucratividade por Bond e Lean (1977) e Schwartzman (1976) em Cool e Schendel (1987).

\section{Tratamento dos Dados}

O método escolhido para o tratamento dos dados foi o hipotético-dedutivo. $\mathrm{O}$ quadro a seguir mostra os passos na realização do tratamento dos dados coletados, bem como descreve os propósitos de cada passo e, suscintamente, os procedimentos estatísticos utilizados. 


\section{Quadro 3: Tratamento dos Dados}

\begin{tabular}{|c|c|c|c|}
\hline Etapas & Procedimento & Objetivo & Método \\
\hline $1^{\mathrm{O}}$ Passo & $\begin{array}{l}\text { Preparação da } \\
\text { Base de Dados }\end{array}$ & $\begin{array}{l}\text { Eliminar quaisquer distorções que } \\
\text { pudessem ocorrer se uma base de } \\
\text { dados de apenas um ano fosse utilizada. }\end{array}$ & $\begin{array}{l}\text { Média dos } 4 \text { anos observados para } \\
\text { cada uma das variáveis, formando assim } \\
11 \text { variáveis estratégic as e } 4 \text { de } \\
\text { desempenho. }\end{array}$ \\
\hline $2^{\mathrm{O}}$ Passo & $\begin{array}{l}\text { Redução das } \\
\text { Variáveis à Forma } \\
\text { Canônica }\end{array}$ & $\begin{array}{l}\text { Fazer com que a distribuição dos } \\
\text { valores de cada empresa dentro das } \\
\text { variáveis se tornem distribuições } \\
\text { homogêneas. }\end{array}$ & $\begin{array}{l}\text { Para cada vetor de variável será } \\
\text { formada uma base canônica, dividindo } \\
\text { o valor da variável de cada empresa } \\
\text { pelo valor da variável de maior } \\
\text { grandeza. }\end{array}$ \\
\hline $3^{\circ}$ Passo & $\begin{array}{l}\text { Teste de } \\
\text { Normalidade }\end{array}$ & $\begin{array}{l}\text { Eliminar da base de dados aquelas } \\
\text { variáveis que possuam distribuição } \\
\text { acentuadamente não-normal e que } \\
\text { poderiam portanto comprometer as } \\
\text { análises da pesquisa. }\end{array}$ & $\begin{array}{l}\text { Observação das mé dias, me dianas, } \\
\text { des vio-padrão, co e ficient e de } \\
\text { assimetria, cutose e visualização gráfica } \\
\text { dos histogramas com curva normal para } \\
\text { cada uma das variáveis. }\end{array}$ \\
\hline $4^{\circ}$ Passo & $\begin{array}{l}\text { Análise de } \\
\text { Correlação }\end{array}$ & $\begin{array}{l}\text { Eliminar da amostra variáveis que } \\
\text { tenham alta correlação entre si }\end{array}$ & $\begin{array}{l}\text { Matrizes de correlação com teste de } \\
\text { hipótese de correlação linear } t w o \text { - } \\
\text { tailed. }\end{array}$ \\
\hline $5^{\circ}$ Passo & Análise de Fatores & $\begin{array}{l}\text { Reduzir o número de variáveis } \\
\text { observáveis a fatores que as } \\
\text { representem. }\end{array}$ & $\begin{array}{l}\text { Análise de fatores com rotação } \\
\text { ottogonal tipo Varimax }\end{array}$ \\
\hline $6^{\circ}$ Passo & Análise de Chuster & $\begin{array}{l}\text { Classificar e agurpar as } 50 \text { empresas da } \\
\text { amostra no espaço es tratégico de } \\
\text { acordo com suas estratégias realizadas. }\end{array}$ & $\begin{array}{l}\text { Análise de Cluster K-means para a } \\
\text { dimensão estratégia. }\end{array}$ \\
\hline $7^{\circ}$ Passo & $\begin{array}{l}\text { Determinação das } \\
\text { estratégias } \\
\text { genéricas de cada } \\
\text { Chuster }\end{array}$ & $\begin{array}{l}\text { Identificar nos } 5 \text { grupos estratégicos } \\
\text { formados as estratégias genéricas de } \\
\text { Poiter. }\end{array}$ & $\begin{array}{l}\text { Análise dos vetores multidimensionais, } \\
\text { comparando os valores que cada fator } \\
\text { assume nos diferentes grupos. }\end{array}$ \\
\hline $8^{\circ}$ Passo & $\begin{array}{l}\text { Verificação da } \\
\text { distinção estatística } \\
\text { dos Chusters }\end{array}$ & $\begin{array}{l}\text { Confirmar se os grupos estratégicos } \\
\text { formados são estatisticamente distintos } \\
\text { entre si }\end{array}$ & $\begin{array}{l}\text { Multivariate Analysis of Variance } \\
\text { (MANOVA). }\end{array}$ \\
\hline $9^{\circ}$ Passo & $\begin{array}{l}\text { Comparação das } \\
\text { médias de } \\
\text { desempenhos } \\
\text { entre grupos } \\
\text { estratégicos }\end{array}$ & $\begin{array}{l}\text { Comprovar as hipóteses de que há } \\
\text { diferenças significativas de desempenho } \\
\text { entre os desempenhos mé dios dos } \\
\text { grupos estratégicos. }\end{array}$ & $\begin{array}{l}\text { Multivariate Analysis of Variance } \\
\text { (MANOVA). }\end{array}$ \\
\hline
\end{tabular}

\section{Apresentação e Análise dos Resultados}

\section{Redução do Espaço Estratégico}

\section{O procedimento de redução do espaço estratégico foi utilizado com o objetivo}


de reduzir o número de variáveis observáveis a fatores que as representem sem perda significativa do seu poder explanatório, assim facilitando a análise.

Para a redução do espaço estratégico, foi realizada uma análise de fatores pelo método dos componentes principais para as 11 variáveis estratégicas (vide Tabela 1).

\section{Tabela 1: Resultado da Análise de Fatores}

\begin{tabular}{|c|c|c|c|c|c|}
\hline VARIÁVEIS & $\begin{array}{l}\text { COMUNALIDADE } \\
\text { INICIAL }\end{array}$ & $\begin{array}{l}\text { COMUNALIDADE } \\
\text { FINAL }\end{array}$ & AUTOVALOR & $\begin{array}{c}\% \\
\text { VARIÂNCIA } \\
\text { EXPLICADA }\end{array}$ & $\begin{array}{c}\% \\
\text { VARIÂNCIA } \\
\text { ACUMULADA }\end{array}$ \\
\hline FOCO & 1 & 0,870 & 1,738 & 15,802 & 55,583 \\
\hline RECEITUÁRIO & 1 & 0,775 & 0,287 & 2,605 & 97,760 \\
\hline GENÉRICO COMMODITY & 1 & 0,913 & 1,135 & 10,318 & 65,901 \\
\hline GENÉRICO DE MARCA & 1 & 0,714 & 0,991 & 9,009 & 74,910 \\
\hline USO CRÔNICO & 1 & 0,699 & 2,466 & 22,421 & 22,421 \\
\hline PROCURA ESPONTÂNEA & 1 & 0,823 & 1,910 & 17,360 & 39,781 \\
\hline NOVOS PRODUTOS & 1 & 0,951 & 0,613 & 5,576 & 88,271 \\
\hline NOVAS MOLÉCULAS & 1 & 0,882 & 0,856 & 7,785 & 82,695 \\
\hline PROMOÇÃO & 1 & 0,860 & 0,366 & 3,324 & 95,155 \\
\hline PREÇO MÉDIO & 1 & 0,780 & 0,392 & 3,560 & 91,831 \\
\hline TAMANHO & 1 & 0,831 & 0,246 & 2,240 & 100,000 \\
\hline
\end{tabular}

Em primeiro lugar, a análise de fatores foi feita sem rotação dos eixos e, em seguida, com rotação ortogonal do tipo varimax, com a intenção de facilitar a identificação dos fatores. A rotação ortogonal facilita essa identificação e conseqüentemente a sua interpretação, pois reduz a carga do primeiro fator e aumenta a carga dos outros fatores em um número menor de variáveis; assim, os fatores, que já possuíam maior carga, ficam ainda mais destacados. Além disso, para permitir uma maior seleção de fatores, considerou-se também como entrada para o procedimento da análise de fatores autovalores maiores ou iguais a 1.0. A análise de fator permitiu a extração de seis fatores que, juntos, explicam 82,69\% das variâncias originais (vide Tabela 2).

\section{Tabela 2: Resultado dos Fatores Extraídos}

\begin{tabular}{|c|c|c|c|c|c|c|}
\hline \multirow{3}{*}{ FATORES } & \multicolumn{3}{|c|}{ SEM ROTAÇÃO ORTOGONAL } & \multicolumn{3}{c|}{ COM ROTAÇÃO ORTOGONAL } \\
\cline { 2 - 7 } & AUTOVALOR & $\begin{array}{c}\text { \% VARLÂNCLA } \\
\text { EXPLICADA }\end{array}$ & $\begin{array}{c}\text { \% VARIÂNCLA } \\
\text { ACUMULADA }\end{array}$ & AUTOVALOR & $\begin{array}{c}\text { \% VARLÂNCIA } \\
\text { EXPLICADA }\end{array}$ & $\begin{array}{c}\text { \% VARLÂNCLA } \\
\text { ACUMULADA }\end{array}$ \\
\hline 1 & 2,466 & 22,421 & 22,421 & 1,921 & 17,461 & 17,461 \\
2 & 1,910 & 17,360 & 39,781 & 1,786 & 16,235 & 33,696 \\
3 & 1,738 & 15,802 & 55,583 & 1,786 & 16,234 & 49,930 \\
4 & 1,135 & 10,318 & 65,901 & 1,373 & 12,481 & 62,411 \\
5 & 0,991 & 9,009 & 74,910 & 1,134 & 10,310 & 72,721 \\
6 & 0,856 & 7,785 & 82,695 & 1,097 & 9,974 & 82,695 \\
\hline
\end{tabular}


Pode-se ver que dois fatores (5 e 6), que possuíam autovalores abaixo de 1 , tiveram as suas cargas aumentadas acima desse valor, após a rotação ortogonal, confirmando, assim, a sua seleção. Na Tabela 3, encontra-se a matriz dos fatores para o espaço estratégico, reduzido após a rotação de eixos. Para facilitar a visualização, somente foram mantidas as cargas com valores maiores que $0.3 \mathrm{em}$ valores absolutos.

Tabela 3: Carga Final dos Fatores após a Rotação

\begin{tabular}{|c|c|c|c|c|c|c|}
\hline \multirow{2}{*}{ VARIÁVEIS } & \multicolumn{6}{|c|}{ FATORES * } \\
\hline & 1 & 2 & 3 & 4 & 5 & 6 \\
\hline $\begin{array}{l}\text { FOCO } \\
\text { RECEITUÁRIO } \\
\text { GENÉRICO COMMODITY } \\
\text { GENÉRICO DE MARCA } \\
\text { USO CRÔNICO } \\
\text { PROCURA ESPONTÂNEA } \\
\text { NOVOS PRODUTOS } \\
\text { NOVAS MOLÉCULAS } \\
\text { PROMOÇÃO } \\
\text { PREÇO MÉDIO } \\
\text { TAMANHO }\end{array}$ & 0,808 & $-0,463$ & $\begin{array}{l}-0,373 \\
0,839 \\
-0,850\end{array}$ & $\begin{array}{c}-0,469 \\
0,587\end{array}$ & 0,961 & 0,909 \\
\hline
\end{tabular}

Só estão representadas as cargas com valores maiores que 0.30 em valores absolutos.

O Quadro 4 apresenta os nomes dos 6 fatores formados a partir das 11 variáveis estratégicas. Esses fatores foram salvos na base de dados e utilizados para formar os grupos estratégicos por meio da análise de cluster.

\section{Quadro 4: Fatores Resultantes da Redução do Espaço Estratégico}

\begin{tabular}{|c|l|l|}
\hline Fator & \multicolumn{1}{|c|}{ Nome do Fator } & \multicolumn{1}{c|}{ Variáveis Agrupadas } \\
\hline 1 & Nível de Diferenciação do Produto & Uso Crônico / Preço Médio \\
\hline 2 & Inovação e Escala & Novas Moléculas / Tamanho da Empresa \\
\hline 3 & Foco no Mercado Ético & Procura Espontânea / Receituário \\
\hline 4 & Nível de Promoção & Genérico de Marca / Promoção \\
\hline 5 & Lançamento de Produtos & Novos Produtos \\
\hline 6 & Genérico Commodity & Genérico Commodity \\
\hline
\end{tabular}




\section{Formação dos Grupos Estratégicos}

Aplicou-se o procedimento de Análise de Cluster K-means no espaço reduzido composto pelos 6 fatores, com o objetivo de agrupar as empresas da amostra de acordo com o elenco de estratégias que utilizem. A premissa por trás desse procedimento é de que dentro de um mesmo cluster, ou grupo estratégico, encontram-se empresas que praticam estratégias competitivas aproximadamente semelhantes entre si; no entanto, muito diferentes de outras empresas fora desse grupo estratégico. Sendo assim, considera-se que as diferenças entre os desempenhos médios das empresas de uma indústria são geradas em função das diferentes decisões estratégicas das empresas, isto é, a forma pela qual cada companhia administra seus recursos (Porter, 1980, 1985).

Na presente pesquisa, foi predeterminado o número de clusters a serem gerados. A escolha de 5 grupos estratégicos reflete a intenção de testar a teoria de Porter (1980, 1985), isto é, dividir as empresas pertencentes à indústria farmacêutica brasileira em 4 grupos que adotam as estratégias genéricas propostas por ele (liderança de custo, diferenciação, enfoque em custo, enfoque em diferenciação), e em um grupo residual chamado de meio-termo ou stuck-in-the-middle. Sendo assim, a montagem da matriz inicial de centros teóricos, isso é, a matriz formada a partir das médias das variáveis de cada empresa que forma os clusters, reflete essa intenção.

A Tabela 4 apresenta a matriz dos centros teóricos informados ao programa, enquanto a Tabela 5 apresenta a matriz dos centróides finais.

Tabela 4: Matriz dos Centróides Iniciais dos 5 Grupos Estratégicos

\begin{tabular}{|l|c|c|c|c|c}
\hline \multirow{2}{*}{ FATORES } & \multicolumn{5}{c}{ CLUSTER } \\
\cline { 2 - 6 } & 1 & 2 & 3 & 4 & 5 \\
\hline Nível de Diferenciação do Produto & $-0,7034$ & 0,4974 & 0,4974 & $-0,7034$ & $-0,2985$ \\
\hline Inovação e Escala & $-0,8233$ & 0,6377 & 0,6377 & $-0,8233$ & $-0,1857$ \\
\hline Foco no Mercado Ético & $-0,3000$ & $-0,3000$ & 0,5412 & 0,5412 & 0,2349 \\
\hline Nível de Promoção & 0,5717 & 0,5717 & $-0,6449$ & $-0,6449$ & $-0,0432$ \\
\hline Lançamento de Produtos & 0,6110 & 0,6110 & $-0,7599$ & $-0,7599$ & $-0,3121$ \\
\hline Genérico Commodity & 0,1169 & $-0,4848$ & $-0,4848$ & 0,1169 & $-0,2713$
\end{tabular}




\section{Tabela 5: Matriz dos Centróides Finais dos 5 Grupos Estratégicos}

\begin{tabular}{|l|c|c|c|c|c}
\hline \multirow{2}{*}{\multicolumn{1}{c|}{ FATORES }} & \multicolumn{5}{c}{ CLUSTER } \\
\cline { 2 - 6 } & 1 & 2 & 3 & 4 & 5 \\
\hline Nível de Diferenciação do Produto & $-0,7128$ & 1,2943 & $-0,3377$ & $-0,4038$ & 0,0061 \\
\hline Inovação e Escala & $-0,8809$ & 0,8698 & 0,3476 & $-0,2538$ & $-0,6008$ \\
\hline Foco no Mercado Ético & $-1,8032$ & $-0,5076$ & 0,3975 & 0,2137 & 0,4266 \\
\hline Nível de Promoção & 0,4016 & 0,1026 & $-0,4416$ & $-1,4174$ & 1,0063 \\
\hline Lançamento de Produtos & $-0,1188$ & 0,4494 & $-0,7255$ & 1,0739 & 0,1877 \\
\hline Genérico Commodity & 0,6116 & $-0,1014$ & $-0,2495$ & 1,2255 & $-0,4044$
\end{tabular}

Neste ponto, cabe comentar as principais alterações ocorridas entre a matriz dos centros teóricos e a matriz dos centros finais dos 5 clusters gerados.

A grande maioria das alterações ocorridas nos valores dos centróides dos clusters não correspondeu a uma modificação da posição relativa entre eles; em outras palavras, na maioria dos casos confirmou-se a matriz inicial. Grupos estratégicos que possuíam como informação inicial uma maior ou menor carga de determinado fator continuaram nessa posição em relação aos outros grupos. Dessa forma, pode-se concluir que a matriz inicial representava adequadamente as opções estratégicas de cada grupo de empresas.

As principais alterações da posição relativa esperada na matriz de centróides iniciais ocorreram no fator 3 (lançamento de produtos). Esperava-se que o cluster 1 juntamente com o 2 fossem os grupos estratégicos com o maior enfoque na estratégia de lançamento de produtos, enquanto os cluster 3 e 4 possuiriam a menor carga desse fator; no entanto a posição relativa dos clusters 1 e 3 se inverteu para esse fator.

Além da alteração no fator 3, podem-se destacar as modificações de posição relativa para o cluster 5 . Na idealização da matriz inicial dos centróides, foram colocadas cargas médias para representar o centro do cluster 5. Com isso, procurava-se a montagem de um grupo estratégico que não possuísse o predomínio de nenhuma estratégia, aqui representadas pelos 6 fatores. Sendo assim, o grupo estratégico formado a partir do cluster 5 seria classificado segundo a tipologia de Porter $(1980,1985)$ para estratégias genéricas como stuck-in-the-middle. A posição relativa de meio-termo para o cluster 5 se confirmou para os fatores 1,2 e 5: nível de diferenciação do produto, inovação e escala e lançamento de produ- 
tos, respectivamente. Já para as fatores 3 e 4 , foco no mercado ético e nível de promoção, a posição relativa do cluster 5 diferiu do esperado.

As 13 empresas que formam o grupo estratégico 5 são, em sua maioria, laboratórios nacionais, salvo algumas empresas multinacionais com participação pouco expressiva no mercado brasileiro. Uma das principais estratégias dos laboratórios nacionais, ao longo dos anos, tem sido lançar medicamentos que se configuram em cópias fiéis de medicamentos de sucesso de empresas multinacionais. Entre as diversas razões para esse tipo de estratégia, podem-se destacar duas mais importantes: (1) as empresas farmacêuticas nacionais não possuem escala para investir em pesquisa; e (2) puderam beneficiar-se da ausência de leis que regulamentassem a propriedade patentária. Essa característica explica a inversão da posição relativa do grupo estratégico 5 para os fatores foco no mercado ético e nível de promoção. O fator nível de promoção foi formado a partir das variáveis genérico de marca e promoção. Logo, possuir alto grau desse fator corrobora a noção intuitiva de que essas empresas praticam as estratégias de cópia, ou, como é chamado na indústria, estratégia de me-too, uma vez que essa é a definição da variável genérico de marca. $\mathrm{O}$ alto grau de investimento em promoção se deve ao fato de o novo produto, isto é, a cópia, concorrer com o produto original lançado anteriormente. O predomínio demostrado pelo cluster 5 no fator 3 - foco no mercado ético - pode ser explicado pelo mesmo raciocínio anterior: uma vez que as empresas possuem recursos escassos, elas procuram lançar os produtos com melhor retorno, isto é, cópias de produtos de sucesso em vendas e que possuam preços mais altos. De forma geral, na indústria farmacêutica os produtos que preenchem esses critérios são os produtos que dependem de prescrição médica. Sendo assim, é fácil entender por que as empresas que praticam a estratégia de cópia possuem alto grau do fator foco no mercado ético e baixo grau no fator genérico commodity. A média de preços praticados no mercado de genérico é muito mais baixa que a média de preços praticada no mercado ético e, além disso, não existe nenhum produto genérico que possa ser considerado sucesso em vendas.

Pelo visto, considerou-se que o foco do grupo estratégico 5 nesses dois fatores não impede a sua classificação segundo a tipologia de Porter $(1980,1985)$ para estratégias genéricas como stuck-in-the-middle, uma vez que o fato de uma empresa possuir um posicionamento classificado como stuck-in-the middle não significa que ela não se utilize de métodos e armas competitivas típicas, mas apenas que a sua estratégia carece de consistência interna como um todo (Dess e Davis, 1984). Nesse caso, a arma competitiva escolhida pelas empresas do grupo 5 foi a estratégia de cópia, porém, certamente, ela carece de solidez. 
Os pontos acima destacados permitem concluir que se pode adotar a classificação inicialmente imaginada para os grupos estratégicos. Assim, Quadro 5 resume a configuração dos 5 grupos estratégicos formados.

\section{Quadro 5: Configuração dos 5 Grupos Estratégicos para o Espaço Reduzido}

\begin{tabular}{|c|c|l}
\hline $\begin{array}{c}\text { Grupo } \\
\text { Estratégico }\end{array}$ & $\begin{array}{c}\text { Número de } \\
\text { Empres as }\end{array}$ & \multicolumn{1}{c}{ Principais Características dos Grupos Estratégicos } \\
\hline 1 & 5 & $\begin{array}{l}\text { Direcionado para estratégia de custos. } \\
\text { Nivel de Diferenciação do Produto e Inovação fracos e Nivel de } \\
\text { Promoção e Genérico Commodity alto. Forte presença no mercado } \\
\text { OTC, dado o fraco Foco no Mercado Ético. }\end{array}$ \\
\hline 2 & 17 & $\begin{array}{l}\text { Direcionado para estratégia de diferenciação. } \\
\text { Forte Diferenciação do Produto, Inovação e Lançamento de Produtos. } \\
\text { Nivel médio de Promoção e ampla presença no mercado OTC, dado o } \\
\text { fraco Foco no Mercado Ético. }\end{array}$ \\
\hline 3 & 6 & $\begin{array}{l}\text { Direcionado para estratégia de enfoque em diferenciação. } \\
\text { Forte nivel de Inovação e Escala e nível médio de Diferenciação do } \\
\text { Produto. Forte Foco no Mercado Ético. }\end{array}$ \\
\hline 5 & 13 & $\begin{array}{l}\text { Direcionado para estratégia de enfoque em custos. } \\
\text { Nivel de Diferenciação do Produto e Inovação fracos e Lançamento de } \\
\text { Produtos e Genérico Commodity altos. Forte Foco no Mercado Etico. }\end{array}$ \\
\hline 5 & $\begin{array}{l}\text { Stuck-in-the-middle. } \\
\text { Atuação fraca na maioria das estratégias, mas com foco na estratégia de } \\
\text { me-too. }\end{array}$ \\
\hline
\end{tabular}

\section{Apresentação dos Desempenhos dos Grupos Estratégicos}

Uma vez definidos os cinco grupos estratégicos pelo procedimento de Análise de Cluster K-means e comprovada a diferença entre seus centros pelo Teste de Hipótese de Hotelling, aplicou-se o procedimento de MANOVA para determinar os desempenhos respectivos, focalizando as 4 variáveis de desempenho identificadas. Com isso procurou-se testar se havia diferença significativa entre as médias das 4 variáveis de desempenho para os 5 grupos estratégicos formados.

Em termos de market share, o melhor desempenho verificado foi para o grupo 3 com pequena diferença em relação ao grupo 2 , enquanto o pior desempenho foi dos grupos 1 e 5 . O grupo 3 também demonstrou o melhor desempenho em termos de market share ponderado. Já os dois grupos com o pior desempenho nessa variável foram os grupos 5 e 4 respectivamente. 


\section{Tabela 6: Estatísticas de Desempenho dos 5 Grupos Estratégicos}

\begin{tabular}{|c|c|c|c|c|}
\hline & NÚMERO DO CLUSTER & MÉDIA & $\begin{array}{l}\text { DESVIO } \\
\text { PADRÃO }\end{array}$ & $\mathrm{N}$ \\
\hline Market Share & $\begin{array}{l}\text { 1- Liderança de Custos } \\
\text { 2- Diferenciação } \\
\text { 3- Enf. em Diferenciação } \\
\text { 4- Enfoque em Custos } \\
\text { 5- Stuck-in-the-middle } \\
\quad \text { Total }\end{array}$ & $\begin{array}{c}0,1189 \\
0,3352 \\
0,3661 \\
0,2151 \\
0,1859 \\
0,27029\end{array}$ & $\begin{array}{c}5,836 \mathrm{E}-02 \\
0,1830 \\
0,2406 \\
0,1313 \\
0,2110 \\
0,2142\end{array}$ & $\begin{array}{c}5 \\
9 \\
17 \\
6 \\
13 \\
50\end{array}$ \\
\hline $\begin{array}{c}\text { Market Share } \\
\text { Ponderado }\end{array}$ & $\begin{array}{l}\text { 1- Liderança de Custos } \\
\text { 2- Diferenciação } \\
\text { 3- Enf. em Diferenciação } \\
\text { 4- Enfoque em Custos } \\
\text { 5- Stuck-in-the-middle } \\
\text { Total }\end{array}$ & $\begin{array}{c}9,613 \mathrm{E}-02 \\
5,540 \mathrm{E}-02 \\
0,1271 \\
3,370 \mathrm{E}-02 \\
4,030 \mathrm{E}-02 \\
7,732 \mathrm{E}-02\end{array}$ & $\begin{array}{c}0,1923 \\
8,030 \mathrm{E}-02 \\
0,2442 \\
3,976 \mathrm{E}-02 \\
0,1243 \\
0,1705\end{array}$ & $\begin{array}{c}5 \\
9 \\
17 \\
6 \\
13 \\
50\end{array}$ \\
\hline $\begin{array}{l}\text { Crescimento de } \\
\text { Market Share }\end{array}$ & $\begin{array}{l}\text { 1- Liderança de Custos } \\
\text { 2- Diferenciação } \\
\text { 3- Enf. em Diferenciação } \\
\text { 4- Enfoque em Custos } \\
\text { 5- Stuck-in-the-middle } \\
\quad \text { Total }\end{array}$ & $\begin{array}{l}0,4214 \\
0,4434 \\
0,3002 \\
0,5752 \\
0,2739 \\
0,3642\end{array}$ & $\begin{array}{l}0,4450 \\
0,2163 \\
0,1428 \\
0,3389 \\
0,2799 \\
0,2681\end{array}$ & $\begin{array}{c}5 \\
9 \\
17 \\
6 \\
13 \\
50\end{array}$ \\
\hline $\begin{array}{l}\text { Crescimento de } \\
\text { Market Share } \\
\text { Ponderado }\end{array}$ & $\begin{array}{l}\text { 1- Liderança de Custos } \\
\text { 2- Diferenciação } \\
\text { 3- Enf. em Diferenciação } \\
\text { 4- Enfoque em Custos } \\
\text { 5- Stuck-in-the-middle } \\
\quad \text { Total }\end{array}$ & $\begin{array}{c}2,177 \mathrm{E}-02 \\
7,423 \mathrm{E}-02 \\
-2,210 \mathrm{E}-02 \\
-0,1824 \\
-7,520 \mathrm{E}-02 \\
-3,340 \mathrm{E}-02\end{array}$ & $\begin{array}{l}0,6169 \\
0,4107 \\
0,3079 \\
0,2595 \\
0,5357 \\
0,4152\end{array}$ & $\begin{array}{c}5 \\
9 \\
17 \\
6 \\
13 \\
50\end{array}$ \\
\hline
\end{tabular}

Quanto à variável crescimento do market share, o melhor desempenho foi alcançado pelo grupo 2, enquanto o pior ficou novamente para o grupo 5. Já com relação à variável crescimento de market share ponderado, os melhores desempenhos foram dos grupos 4 e 2; o grupo 5 se confirmou como o pior desempenho, também nessa variável.

Para confirmar se as médias dos desempenhos de cada grupo estratégico eram estatisticamente diferentes, também foi utilizado o Teste de Hipótese de Hotelling. A Tabela 7 apresenta o resultado do teste, utilizando um nível de significância de 5\%. 


\section{Tabela 7: Teste de Significância Multivariável de Hotelling para a Comparação de Desempenho}

\begin{tabular}{|c|c|c|c|c|c|c|c|}
\hline & & & \multicolumn{5}{|c|}{ HYPOTHESIS } \\
\hline & & & VALUE & $\mathrm{F}$ & df & ERROR df & sig. \\
\hline \multirow{8}{*}{ Effect } & \multirow{4}{*}{ INTERCEPT } & Pillai's Trace & 0,869 & $69,669^{b}$ & 4,000 & 42,000 & 0,000 \\
\hline & & Wilks'Lambda & 0,131 & $69,669^{b}$ & 4,000 & 42,000 & 0,000 \\
\hline & & Hotelling's Trace & 6,635 & $69,669^{\mathrm{b}}$ & 4,000 & 42,000 & 0,000 \\
\hline & & Roy's Largest Root & 6,635 & $69,669^{b}$ & 4,000 & 42,000 & 0,000 \\
\hline & \multirow{4}{*}{ QC1_1 } & Pillai's Trace & 0,595 & 1,964 & 16,000 & 180,000 & 0,018 \\
\hline & & Wilks'Lambda & 0,498 & 2,066 & 16,000 & 128,950 & 0,014 \\
\hline & & Hotelling's Trace & $\mathbf{0 , 8 3}$ & 2,102 & 16,000 & 162,000 & 0,010 \\
\hline & & Roy's Largest Root & 0,545 & 6,131 & 4,000 & 45,000 & 0,001 \\
\hline
\end{tabular}

O teste apresentou nível de significância de 0,010 , o que nos permite concluir que as médias de desempenho, medidas em função das 4 variáveis apresentadas, são diferentes entre si para os 5 grupos estratégicos, pelo que confirmamos a hipótese de Porter $(1980,1985)$ : ele defende que os grupos estratégicos de uma dada indústria apresentam diferentes desempenhos. Em outras palavras, a média dos desempenhos das empresas, que formam um grupo estratégico de dada indústria, é estatisticamente diferente das médias dos desempenhos das empresas que formam os outros grupos estratégicos da mesma indústria.

\section{Interpretação dos Resultados à luz da Teoria de Porter}

\section{Análise da Formação dos Grupos Estratégicos}

A Figura 1 apresenta as empresas que formam cada grupo estratégico e resume a configuração dos grupos estratégicos segundo a teoria de Porter (1980, 1985). 


\section{Figura 1: Configuração dos Grupos Estratégicos à Luz da Tipologia de Porter}

\begin{tabular}{|c|c|}
\hline GRUPO 1 & LIDERANÇA DE CUSTOS \\
\hline $\begin{array}{l}\text { D M IND. FTCA } \\
\text { NQ-SIGMA PHARMA } \\
\text { PROCTER GAMBLE } \\
\text { S.K. BEECHAM CONSUM } \\
\text { VIRTUS }\end{array}$ & \\
\hline
\end{tabular}

\begin{tabular}{|l|}
\hline \multicolumn{1}{|c|}{ GRUPO 2} \\
\hline ASTRA BRASI \\
BAYER \\
BOEHRINGER ING \\
BRISTOL MYER SQUIB \\
KNOLL \\
MERCK SHARP DOHME \\
PFIZER \\
SCHERING DO BRASL \\
ZENECA \\
\hline
\end{tabular}

\begin{tabular}{|ll|}
\hline \multicolumn{1}{|c|}{ GRUPO 3 } & \multicolumn{1}{c|}{$\begin{array}{c}\text { ENFOQUE EM } \\
\text { DIFERENCIAÇÃO }\end{array}$} \\
\hline ABBOTT & ORGANON \\
ALLERGAN & RHODIA \\
ASTA MEDICA & ROCHE \\
BYK & S.K. BEECHAM PHARMA \\
HOECHST MAROUSSEL & SANOFIWINTHROP \\
JANSSEN CILAG & SCHERING PLOUGH \\
LILLY & SERVIER DO BRASIL \\
MERCK & ZAMBON \\
NOVARTIS & \\
\hline
\end{tabular}

\begin{tabular}{|c|c|}
\hline GRUPO 4 & ENFOQUE EM CUSTOS \\
\hline $\begin{array}{l}\text { ALCON } \\
\text { GALDERMA } \\
\text { GLAXO WELLCOME } \\
\text { STIEFEL } \\
\text { WHITEHALL } \\
\text { WYETH }\end{array}$ & \\
\hline
\end{tabular}

\begin{tabular}{|ll|}
\hline \multicolumn{1}{|c|}{ GRUPO 5} & STUCK IN THE MIDDLE \\
\hline ACHE & MARJAN \\
BALDACCI & MEDLEY \\
BIOSINTETICA & PHARMACLA UPJOHN \\
EUROFARMA & PRODOME \\
FARMALAB IQF & SEARLE DO BRASI \\
FARMASA & SINTOFARMA \\
LIBBS & \\
\hline
\end{tabular}

\section{Grupo 1 - Liderança de Custos}

O grupo estratégico 1, classificado segundo a tipologia proposta por Porter $(1980,1985)$ como liderança de custo, é formado por 5 empresas da amostra (vide Tabela 8). A sua principal característica é o baixo grau dos fatores 1 e 2 , nível de diferenciação do produto e inovação e escala, respectivamente.

Outra característica que podemos destacar é a forte presença no mercado OTC, devido ao baixo grau do fator foco no mercado ético, do grupo 1 como um todo. A única exceção que devemos citar é referente à empresa Neo-Química Sigma Pharma que possui alto grau desse fator, 0.760 . O foco no mercado ético demonstrado pela Neo-Química pode ser explicado por seu alto grau do fator 6: essa empresa possui o maior grau do fator genérico commodity entre todas as 
empresas da amostra, 4,217. Existe forte correlação entre o foco no mercado ético e genérico, devido ao fato de quase a totalidade dos lançamentos de produtos genéricos serem medicamentos que necessitam de prescrição médica. Ainda sobre o fator 3, pode-se destacar que o grupo 1 possui as duas empresas com $o$ menor foco no mercado ético, a Procter \& Gamble $(-3,142)$ e a divisão de consumo da SmithKline Beecham $(-3,418)$. As duas empresas possuem diversos produtos OTC, conhecidos pelo público em geral. A Procter \& Gamble, por exemplo, é dona das marcas Hipoglos e Vick, enquanto a SK Beecham Consumo possui uma série de medicamentos conhecidos como o Sal de Eno, Sonrisal, Leite de Magnésia de Phillips, Emulsão Scott, Sal de Andrews, entre outros.

\section{Tabela 8: Empresas do Grupo Estratégico 1}

\begin{tabular}{|l|c|c|c|c|c|c}
\hline EMPRESAS & $\begin{array}{c}\text { NIVEL DE } \\
\text { DIFERENCIAÇO } \\
\text { DO PRODUTO }\end{array}$ & $\begin{array}{c}\text { INOVACÃO E } \\
\text { ESCALA }\end{array}$ & $\begin{array}{c}\text { FOCO NO } \\
\text { MERCADO } \\
\text { ETICO }\end{array}$ & $\begin{array}{c}\text { NIVEL DE } \\
\text { PROMOÇÃO }\end{array}$ & $\begin{array}{c}\text { LANÇAMENTO } \\
\text { DE PRODUTOS }\end{array}$ & $\begin{array}{c}\text { GENÉRICO } \\
\text { COMMODTY }\end{array}$ \\
\hline D M IND. FTCA & $-0,605$ & $-0,574$ & $-1,646$ & 0,075 & $-0,255$ & $-0,410$ \\
\hline NQ-SIGMA PHARMA & $-0,599$ & $-1,239$ & 0,760 & 2,241 & 0,229 & 4,217 \\
\hline PROCTER GAMBLE & $-1,058$ & $-0,749$ & $-3,142$ & $-0,645$ & 0,845 & $-0,590$ \\
\hline S.K. BEECHAM CONSUM & $-0,298$ & $-0,978$ & $-3,418$ & 0,558 & $-0,634$ & 0,345 \\
\hline VIRTUS & $-1,003$ & $-0,865$ & $-1,570$ & $-0,221$ & $-0,779$ & $-0,503$
\end{tabular}

\section{Grupo 2 - Diferenciação}

O grupo estratégico 2, classificado segundo a teoria de Porter $(1980,1985)$ como grupo que pratica a estratégia de diferenciação, é formado por 9 empresas da amostra (vide Tabela 9), todas elas de capital estrangeiro. A sua principal característica é o alto grau dos fatores nível de diferenciação do produto e inovação e escala.

Quanto ao fator nível de diferenciação do produto, pode-se destacar a empresa Merck Sharp and Dohme com o maior grau desse fator entre as empresas da amostra $(3,209)$. Isso pode ser explicado pelo fato de ser esse fator formado a partir das variáveis uso crônico e preço médio, e de a Merck Sharp possuir o maior preço médio de todas as empresas da amostra. A empresa Zeneca, por sua vez, também pertencente ao grupo estratégico 2, possui o segundo maior grau do fator nível de diferenciação do produto $(2,141)$, devido a fato de ser a empresa com o maior grau da variável uso crônico.

Quanto ao fator inovação e escala, pode-se destacar a empresa Pfizer com o maior grau desse fator entre as empresas da amostra $(2,263)$. A Pfizer é a empresa que tem o maior percentual do seu faturamento advindo de lançamentos de 
inovações, isso é, possui o maior grau da variável novas moléculas. Além disso, a Pfizer também se destaca por possuir os maiores graus dos fatores nível de promoções e lançamento de produtos entre as empresas do seu grupo estratégico.

Tabela 9: Empresas do Grupo Estratégico 2

\begin{tabular}{|c|c|c|c|c|c|c|}
\hline EMPRESAS & $\begin{array}{c}\text { NIVEL DE } \\
\text { DIFERENCIAÇÃO } \\
\text { DO PRODUTO }\end{array}$ & $\begin{array}{l}\text { INOVAÇÃO E } \\
\text { ESCALA }\end{array}$ & $\begin{array}{c}\text { FOCO NO } \\
\text { MERCADO } \\
\text { ÉTICO }\end{array}$ & $\begin{array}{l}\text { NIVEL DE } \\
\text { PROMOÇÃO }\end{array}$ & $\begin{array}{l}\text { LANÇAMENTO } \\
\text { DE PRODUTOS }\end{array}$ & $\begin{array}{l}\text { GENÉRICO } \\
\text { COMMODITY }\end{array}$ \\
\hline ASTRA BRASI & 1,384 & 0,276 & 0,044 & 0,293 & $-0,017$ & 0,323 \\
\hline$B A Y E R$ & 1,242 & 0,430 & $-1,326$ & $-0,040$ & $-0,379$ & $-0,091$ \\
\hline BOEHRINGER ING & $-0,466$ & 1,424 & $-1,281$ & 0,599 & 1,608 & $-0,704$ \\
\hline BRISTOL MYER SQUIB & 0,279 & 1,721 & $-0,210$ & 0,193 & 0,611 & $-0,276$ \\
\hline KNOLL & 0,453 & 0,393 & $-1,350$ & 0,172 & 0,102 & $-0,049$ \\
\hline MERCK SHARP DOHME & 3,209 & 0,543 & 0,459 & $-0,287$ & 0,093 & $-0,528$ \\
\hline PFIZER & 1,502 & 2,263 & 0,284 & 1,013 & 1,548 & 0,031 \\
\hline SCHERING DO BRASI & 1,906 & 1,145 & $-1,115$ & $-0,527$ & 0,962 & 0,352 \\
\hline ZENECA & 2,141 & $-0,368$ & $-0,073$ & $-0,492$ & $-0,483$ & 0,030 \\
\hline
\end{tabular}

Com relação ao fator 3 , o grupo 2 como um todo possui baixo foco no mercado ético, ou seja, não atua com estratégia de foco, mas com estratégia de escopo amplo. Quanto a esse fator, podem-se destacar as empresas Bayer $(-1,326)$, Boehringer $(-1,281)$ e Knoll $(-1,350)$, por possuírem os menores graus desse fator entre as empresas do seu grupo estratégico. Assim como para as empresas do grupo 1 podem-se citar alguns medicamentos que conferem a essas empresas o baixo foco no mercado ético: Aspirina no caso da Bayer; Anador, Buscopan e Mucosolvan para a Boehringer, e a Neosaldina para a Knoll, cada um desses medicamentos representando grande fatia do faturamento dessas empresas.

\section{Grupo 3 - Enfoque em Diferenciação}

O grupo estratégico 3, classificado segundo a tipologia proposta por Porter (1980, 1985) como direcionado à estratégia de enfoque em diferenciação, é o maior grupo entre os cinco grupos estratégicos da pesquisa e formado por 17 empresas da amostra (vide Tabela 10), todas elas de capital estrangeiro. As suas principais características são o forte nível de inovação e escala e grande foco no mercado ético. 
Tabela 10: Empresas do Grupo Estratégico 3

\begin{tabular}{|c|c|c|c|c|c|c|}
\hline EMPRESAS & $\begin{array}{c}\text { NÍVEL DE } \\
\text { DFERENCIAÇÄO } \\
\text { DO PRODUTO }\end{array}$ & $\begin{array}{c}\text { INOVAÇÃO E } \\
\text { ESCALA }\end{array}$ & $\begin{array}{c}\text { FOCONO } \\
\text { MERCADO } \\
\text { ÉTICO }\end{array}$ & $\begin{array}{l}\text { NÍVEL DE } \\
\text { PROMOÇÄO }\end{array}$ & $\begin{array}{l}\text { LANÇAMENTO } \\
\text { DE PRODUTOS }\end{array}$ & $\begin{array}{l}\text { GENERICO } \\
\text { COMMODITY }\end{array}$ \\
\hline ABBOTT & $-0,521$ & 0,288 & 0,126 & 0,572 & $-0,481$ & $-0,482$ \\
\hline ALLERGAN & $-0,744$ & $-0,746$ & 0,444 & $-1,138$ & $-0,271$ & $-0,330$ \\
\hline ASTA MEDICA & $-0,763$ & $-0,076$ & 0,517 & 0,123 & $-1,190$ & $-0,656$ \\
\hline BYK & $-0,433$ & 0,244 & $-0,056$ & $-0,310$ & $-0,780$ & $-0,415$ \\
\hline HOECHST MA ROUSSEL & $-0,855$ & 2,092 & $-0,076$ & $-0,197$ & $-0,638$ & $-0,778$ \\
\hline JANSSEN CILAG & $-0,703$ & 1,153 & 0,541 & $-0,346$ & $-0,522$ & $-0,759$ \\
\hline LILLY & 0,554 & 0,519 & 0,205 & $-0,994$ & 0,249 & 0,190 \\
\hline$M E R C K$ & $-0,300$ & $-0,296$ & $-0,047$ & $-0,046$ & $-0,760$ & $-0,267$ \\
\hline NOVARTIS & $-0,294$ & 1,592 & 0,343 & $-0,594$ & $-0,473$ & 0,217 \\
\hline ORGANON & 0,497 & 0,108 & $-0,382$ & $-0,949$ & $-0,295$ & 0,059 \\
\hline RHODIA & $-1,347$ & 0,578 & 1,607 & $-0,037$ & $-0,613$ & $-0,485$ \\
\hline ROCHE & $-0,541$ & 0,638 & 0,654 & $-0,694$ & $-0,929$ & 0,620 \\
\hline S.K. BEECHAM PHARMA & $-0,241$ & 0,305 & 0,889 & $-0,535$ & $-1,412$ & $-0,136$ \\
\hline SANOFI WINTHROP & $-0,946$ & 0,756 & 0,025 & $-0,300$ & $-0,850$ & $-0,804$ \\
\hline SCHERNG PLOUGH & $-0,778$ & 0,690 & 0,586 & $-0,823$ & $-1,199$ & $-0,193$ \\
\hline SERV ER DO BRASL & 1,902 & $-1,382$ & 0,857 & $-1,445$ & $-1,1074$ & $-0,139$ \\
\hline ZAMBON & $-0,228$ & $-0,551$ & 0,523 & 0,206 & $-1,095$ & 0,117 \\
\hline
\end{tabular}

Podem-se destacar as empresas Hoechst e Novartis pelo grau do fator inovação e escala, 2,092 e 1,592, respectivamente. A razão disso está, principalmente, na escala da operação, pois a Novartis é a maior empresa do mercado, enquanto a Hoechst é a terceira pelo critério de faturamento.

Quanto ao foco no mercado ético, podem-se destacar as empresas Janssen Cilag, Rhodia, Roche, SmithKline Pharma, Schering Plough, Servier e Zambon como as empresas desse grupo estratégico com os maiores graus desse fator.

Com relação aos fatores nível de promoção, lançamento de produtos e genérico commodity, o grupo 3 apresentou grande homogeneidade entre as empresas, a grande maioria possuindo baixo grau desses três fatores.

\section{Grupo 4 - Enfoque em Custos}

O grupo estratégico 4, classificado segundo a tipologia proposta por Porter $(1980,1985)$ como direcionado à estratégia de enfoque em custos, é formado por 
6 empresas da amostra (vide Tabela 11). Suas principais características são o fraco nível de diferenciação do produto e de inovação e escala.

Tabela 11: Empresas do Grupo Estratégico 4

\begin{tabular}{|c|c|c|c|c|c|c|}
\hline EMPRESAS & $\begin{array}{c}\text { NIVEL DE } \\
\text { DIFERENCIAÇÃO } \\
\text { DO PRODUTO }\end{array}$ & $\begin{array}{l}\text { INOVAÇÃO E } \\
\text { ESCALA }\end{array}$ & $\begin{array}{c}\text { FOCO NO } \\
\text { MERCADO } \\
\text { ETICO }\end{array}$ & $\begin{array}{c}\text { NÍVEL DE } \\
\text { PROMOÇÃO }\end{array}$ & $\begin{array}{l}\text { LANÇAMENTO } \\
\text { DE PRODUTOS }\end{array}$ & $\begin{array}{l}\text { GENÉRICO } \\
\text { COMMODITY }\end{array}$ \\
\hline ALCON & $-0,428$ & $-1,141$ & 0,652 & $-2,391$ & 1,189 & $-0,073$ \\
\hline GALDERMA & $-0,575$ & $-1,598$ & 0,842 & $-2,188$ & 2,062 & 0,030 \\
\hline GLAXO WELLCOME & $-0,455$ & 1,040 & 0,596 & $-0,696$ & $-0,258$ & 2,226 \\
\hline STIEFEL & 0,031 & $-0,917$ & 0,072 & $-1,280$ & 2,443 & $-0,106$ \\
\hline WHITEHALL & $-0,930$ & $-0,342$ & $-0,721$ & $-1,191$ & $-0,554$ & 2,574 \\
\hline WYETH & $-0,066$ & 1,435 & $-0,159$ & $-0,758$ & 1,562 & 2,701 \\
\hline
\end{tabular}

De forma geral, o grupo é muito homogêneo quanto ao fator 1; no entanto podem-se destacar as empresas Glaxo Wellcome e Wyeth como exceções no que diz respeito ao fator inovação e escala. Diferentes do resto das empresas do grupo, essas duas possuem grau relativamente alto desse fator, devido principalmente à variável tamanho.

O grupo 4 como um todo também possui alto grau do fator 3 , foco no mercado ético; no entanto podem-se citar as empresas Whitehall e Wyeth como exceções. No caso da Whitehall, o seu baixo grau do fator 3 pode ser explicado pela alta participação de produtos tais como Stresstabs, Centrum, Mucofan, Epocler e Magnésia Bisurada em seu faturamento total. Já no caso da Wyeth, o motivo se deve ao alto grau de procura espontânea. Apesar de a linha de produtos da Wyeth ser composta basicamente de produtos éticos, ela possui alto índice de procura espontânea, devido à repetição de receitas. Isso acontece porque a empresa possui como os seus principais produtos os contraceptivos, tais como as pílulas Minulet, Nordette e Harmonet, muito propensos à repetição de receitas.

Além disso, o grupo 4 também pode ser considerado um grupo com grande foco em classes terapêuticas, isto é, grande parte do faturamento das empresas Alcon, Galderma, Stiefel e Wyeth vem de diversos medicamentos da principal classe terapêutica de cada laboratório. A Alcon, por exemplo, possui 100\% do seu faturamento proveniente de uma mesma classe terapêutica, classe S - órgãos do sensório. A Galderma, por sua vez, também tem todo o seu faturamento de uma única classe terapêutica, a classe $\mathrm{D}$ - produtos dermatológicos. Já a Stiefel e a Wyeth possuem quase a totalidade do seu faturamento proveniente de produtos das classes D - produtos dermatológicos, no caso da Stiefel, e G sistema genitor, no caso da Wyeth. 
Quanto aos fatores lançamento de produtos e genérico commodity, o grupo 4, de forma geral, demostrou possuir relativo direcionamento para essas estratégias. Podem-se destacar a empresa Galderma com um alto grau (2.062) do fator lançamento de produtos e as empresas Glaxo Wellcome e Wyeth, com grande participação do mercado de genéricos commodities. Quanto à Glaxo, é importante destacar que a sua participação expressiva no mercado de genéricos pode não representar uma estratégia da empresa, pois se deve a um único produto, a Digoxina, com a sua venda direcionada principalmente a hospitais.

\section{Grupo 5 - Stuck-in-the-middle}

O grupo estratégico 5, classificado segundo a tipologia proposta por Porter $(1980,1985)$ como stuck-in-the-middle, é formado por 13 empresas da amostra (vide Tabela 12).

\section{Tabela 12: Empresas do Grupo Estratégico 5}

\begin{tabular}{|c|c|c|c|c|c|c|}
\hline EMPRESAS & $\begin{array}{c}\text { NÍVEL DE } \\
\text { DIFERENCIAÇÃO } \\
\text { DO PRODUTO }\end{array}$ & $\begin{array}{l}\text { INOVAÇÃO E } \\
\text { ESCALA }\end{array}$ & $\begin{array}{c}\text { FOCO NO } \\
\text { MERCADO } \\
\text { ÉTICO }\end{array}$ & $\begin{array}{c}\text { NIVEL DE } \\
\text { PROMOÇÃO }\end{array}$ & $\begin{array}{l}\text { LANÇAMENTO } \\
\text { DE PRODUTOS }\end{array}$ & $\begin{array}{l}\text { GENERICO } \\
\text { COMMODITY }\end{array}$ \\
\hline $\mathrm{ACHE}$ & $-1,191$ & 1,122 & 0,328 & 1,285 & 0,512 & $-1,336$ \\
\hline$B A L D A C C I$ & 1,061 & $-1,615$ & $-0,252$ & 0,166 & $-1,048$ & $-0,227$ \\
\hline BIOSINTEIICA & 1,664 & $-1,040$ & 0,031 & 1,472 & 0,447 & $-0,368$ \\
\hline EUROFARMA & $-0,214$ & $-0,485$ & $-0,186$ & 0,846 & $-0,329$ & $-0,469$ \\
\hline FARMALAB IQF & 0,415 & $-1,275$ & 0,477 & 1,190 & $-0,924$ & 0,421 \\
\hline FARMASA & $-0,499$ & $-0,500$ & $-0,211$ & 0,563 & $-0,453$ & $-0,256$ \\
\hline $\mathrm{LBBBS}$ & 0,795 & $-0,938$ & 0,511 & 1,419 & $-0,187$ & $-0,428$ \\
\hline MARJAN & $-0,606$ & $-1,402$ & 1,039 & $-0,135$ & 1,490 & $-0,931$ \\
\hline MEDLEY $Y$ & $-0,862$ & $-0,390$ & 0,265 & 1,810 & 1,254 & $-0,167$ \\
\hline PHARMACLA UPJOHN & $-0,133$ & $-0,802$ & 1,905 & 0,122 & 1,420 & $-1,352$ \\
\hline PRODOME & $-0,726$ & $-0,408$ & 0,430 & 0,925 & $-1,343$ & $-0,455$ \\
\hline SEARLE DO BRASL & $-0,212$ & 0,390 & 0,892 & 1,311 & $-0,356$ & 0,900 \\
\hline SINTOFARMA & $-0,864$ & $-0,467$ & 0,318 & 2,107 & 1,956 & $-0,589$ \\
\hline
\end{tabular}

De forma geral, o grupo 5 parece não apresentar homogeneidade entre as empresas que o compõem em nenhum dos 5 fatores. As empresas pertencentes a esse grupo demonstram fraca atuação na maioria das estratégias representadas pelos fatores; no entanto, como se discutiu anteriormente, focam os seus esforços na estratégia de cópia de medicamentos de sucesso. 
Como exemplo, pode-se citar a empresa Aché, maior laboratório nacional, e que ocupou a segunda colocação na classificação nacional segundo o critério de faturamento entre os anos de 1995 a 1998. Segundo a Gazeta Mercantil (1997), o Grupo Aché registrou, antes da aprovação da Lei de Patentes (1997), isto é, antes do início da necessidade de pagamento de royalties para as empresas descobridoras do medicamento, um número de produtos suficiente para garantir o faturamento de 5 anos, com destaque para produtos de maior venda como antihipertensivos, vitamínicos e anti-reumáticos.

Antes da Lei de Patentes, a maioria dos laboratórios farmacêuticos nacionais, assim como o Grupo Aché, possuía como a sua principal estratégia de lançamentos de novos produtos a cópia de medicamentos de grande venda; no entanto, desde 1997, com o impedimento legal desse mecanismo, esses laboratórios procuram outras formas de competir no mercado farmacêutico.

Como conclusão, pode-se dizer que as empresas pertencentes ao grupo estratégico 5 ainda não possuem estratégia consistente; podem assim ser classificadas como stuck-in-the-middle.

\section{Comparaçăo dos Desempenhos dos Grupos Estratégicos}

A Figura 2 apresenta uma comparação gráfica entre os desempenhos dos cinco grupos estratégicos para cada uma as variáveis de desempenho.

\section{Figura 2: Comparação entre os Desempenhos dos Grupos Estratégicos}
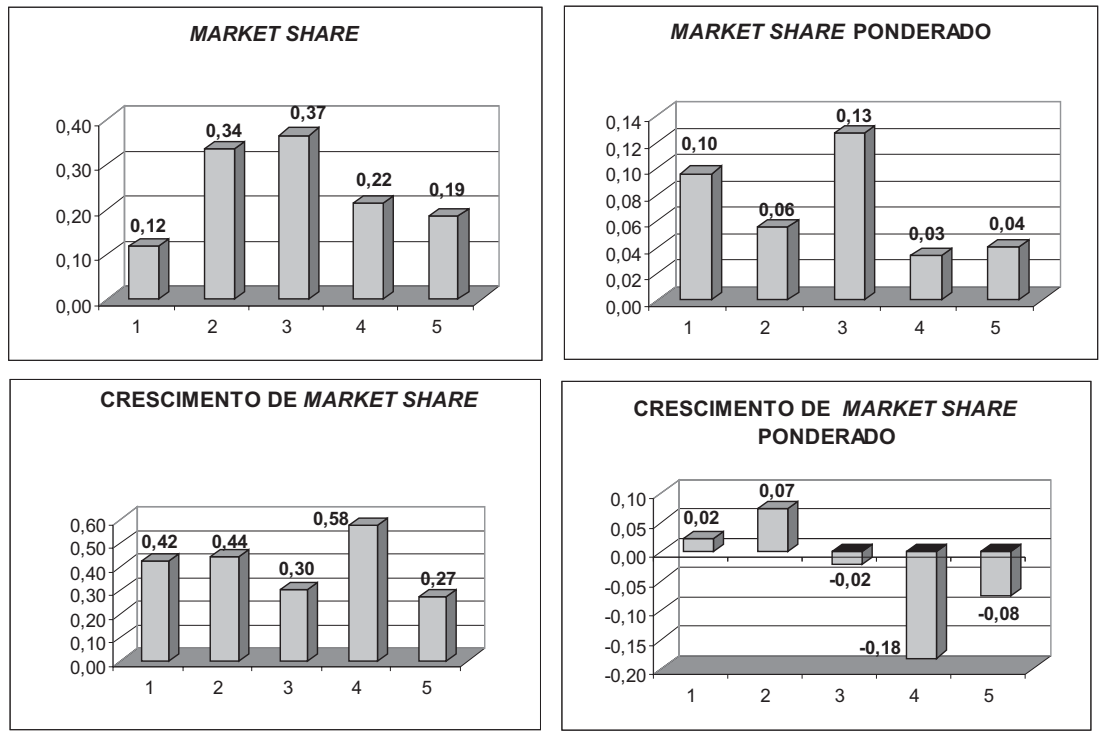
Utilizando a comparação feita na Figura 2, podem-se destacar três pontos principais.

Pode-se indicar uma tendência de melhor desempenho, considerando as variáveis utilizadas no estudo, para grupos que pratiquem estratégia de diferenciação. Os grupos 2 e 3, classificados segundo a tipologia proposta por Porter $(1980,1985)$ como grupos que praticam estratégia de diferenciação ampla e enfoque em diferenciação, respectivamente, auferiram os melhores desempenhos entre os cinco grupos estratégicos do estudo. O grupo 3 alcançou o melhor desempenho nas variáveis market share e market share ponderado, enquanto o grupo 2 ficou com o melhor desempenho na variável crescimento de market share ponderado. É importante destacar, ainda, que o grupo 2 - diferenciação ampla - auferiu excelente desempenho em todas as variáveis, tendo alcançado, além do melhor desempenho na variável crescimento de market share ponderado, o segundo melhor desempenho nas variáveis market share e crescimento do market share e o terceiro melhor desempenho na variável restante - market share ponderado - o que parece fazer dele, de forma geral, o grupo estratégico com o melhor desempenho entre todos.

A única ressalva que se deve fazer nesse ponto diz respeito ao desempenho alcançado pelo grupo estratégico 3 - enfoque em diferenciação - nas variáveis que medem crescimento. $\mathrm{O}$ seu desempenho não foi satisfatório nessas variáveis, devido ao fato de ser composto por empresas que possuem grande participação de mercado, como as empresas Novartis, Hoechst, Roche e Janssen Cilag, respectivamente, $1^{\mathrm{a}}, 3^{\mathrm{a}}, 5^{\mathrm{a}}$ e $7^{\mathrm{a}}$ maiores empresas do mercado. Dado que as variáveis de crescimento são medidas em base percentual sobre o market share do período anterior, é muito difícil que empresas que possuam grande market share cresçam às mesmas taxas que empresas que possuem pequena participação de mercado. Esse efeito, no entanto, é menor para as empresas do grupo estratégico 2 - estratégia de diferenciação ampla - as quais, apesar de possuírem bom desempenho nas variáveis market share e market share ponderado, também demonstraram bom desempenho nas variáveis de crescimento.

- Os grupos estratégicos 1 e 4, classificados segundo a tipologia proposta por Porter $(1980,1985)$ como grupos que praticam estratégia de liderança de custos e enfoque em liderança de custos, respectivamente, não alcançaram, de forma geral, bom desempenho nas variáveis market share e market share ponderado; entretanto essa situação se modifica, quando se analisam as variáveis de crescimento. $\mathrm{O}$ grupo 4 - enfoque em liderança de custos - auferiu o melhor desempenho na variável crescimento de market share ponderado, enquanto o grupo 1 - liderança de custos - ficou com os segundo e o terceiro melhor desempenho nas variáveis crescimento de market share e crescimento 
de market share ponderado. Podemos verificar assim uma possível tendência, que associa melhores desempenhos em variáveis de crescimento em grupos que pratiquem estratégia de custo.

- Quanto ao grupo estratégico 5, classificado segundo a tipologia proposta por Porter $(1980,1985)$ como meio-termo ou sem posicionamento estratégico, podem-se destacar os piores desempenhos dos cinco grupos estratégicos da pesquisa. O grupo 5 obteve o pior desempenho nas variáveis crescimento de market share e crescimento de market share ponderado, enquanto nas variáveis market share e market share ponderado ficou com o segundo pior desempenho. A partir dessa constatação, pode-se concluir que foram confirmadas as previsões de Porter (1985). Para as empresas que se fixam no meio-termo é quase certa uma posição de desvantagem em relação aos concorrentes, isto é, obtêm desempenho abaixo da média do mercado (Porter, 1985).

\section{Conclusão}

Com relação à tipologia proposta por Porter $(1980,1985)$, os resultados obtidos permitem concluir que o modelo se mostrou, sob os aspectos descritivo e explicativo, adequado à análise da indústria farmacêutica brasileira. Os procedimentos de análise de fatores principais e análise de clusters forneceram subsídios para o mapeamento das estratégias competitivas relevantes para a indústria farmacêutica, geraram grupos estratégicos coerentes com a realidade do mercado brasileiro, bem como puderam identificar as posturas competitivas das empresas que os compõem.

Além disso, o modelo demonstrou possuir poder de explicação, ao relacionar a pertinência a um determinado grupo estratégico com o desempenho das empresas do mercado farmacêutico brasileiro, confirmando até mesmo a hipótese básica do modelo de Porter $(1980,1985)$ : assevera que a empresa que fixa a estratégia por ele denominada stuck-in-the-middle está fadada a atingir desempenhos piores de mercado que as empresas que praticam uma das estratégias genéricas descritas em sua tipologia.

Quanto ao desempenho dos grupos estratégicos 1 e 4 - liderança de custos e enfoque em custos - pôde-se constatar que as empresas que praticam estratégias ligadas a custos não demonstraram bom desempenho nas variáveis que medem participação de mercado (market share e market share ponderado); no entanto demonstraram ótimo desempenho na variável crescimento de market share. 
Pôde-se indicar, ainda, a tendência de melhor desempenho de um grupo em relação a outro em função da estratégia de diferenciação. Os grupos estratégicos que praticaram as estratégias de diferenciação ampla e enfoque em diferenciação, grupos 2 e 3 respectivamente, sem dúvida auferiram, de forma geral, os melhores resultados nas quatro variáveis destinadas a medir os desempenhos das empresas contidas na pesquisa. Esse desempenho superior das empresas que praticam estratégias de diferenciação, sem dúvida, se deve ao enfoque em fatores tais como: (1) nível de diferenciação do produto, que engloba as variáveis preço médio e uso crônico; e (2) inovação e escala que, por sua vez, engloba as variáveis novas moléculas e tamanho da empresa.

\section{BibliografiA}

BOGNER, W. C. ;

THOMAS, H.;

MCGEE, J.

A longitudinal study of competitive positions and entry paths of european firms in the U.S. pharmaceutical market. Strategic Management Journal, v. 17, p. 85-107, 1996.

COOL, K.;

DIERICKX, I.

Rivalry, strategic groups and firm profitability. Strategic Management Journal, v. 14, p. 47-59, 1993.

COOL, K.;

SCHENDEL, D.

Strategic group formation and performance: the case of the U.S. pharmaceutical industry, 1963 - 1982. Management
Science, v. 33, n. 9, p.1102-1124, Sept. 1987.

DESS, G. G.;

DAVIS, P. S.

Porter's (1980) generic strategies as determinants of strategic group membership and organizational performance. Academy of Management Journal, v. 27, n. 3, p. 467-488, Sept. 1984.

GAZETA MERCANTIL.

A indústria farmacêutica. Panorama Setorial, agosto, 1997.

HAMBRICK, D. C.

High profit strategies in mature capital goods industries: a contingency approach. Academy of Management Journal, v. 26, n. 4, p. 687-707, 1983a. 
Some tests of the effectiveness and functional attributes of Miles and Snow's strategic types. Academy of Management Journal, v. 26, n. 1, p. 5-26, 1983 b.

HILL, C. W.

Differentiation versus low cost or differentiation and low cost: a contingency framwork. Academy of Management Review, v. 13, n. 3, p. 401-412, 1988.

HILL, C. W.;

DEEDS, D. L.

The importance of industry structure for the determination of the firm profitability: a neo-austrian perspective. Journal of Management Studies, v. 33, n. 4, p. 429-451, July 1996.

KIM, L.;

LIM, Y.

Environment, generic strategies and performance in a rapidly developing country: a taxonomic approach. Academy of Management Journal, v. 31, p. 802-827, 1988.

KOTHA, S.;

VADLAMANI, B. L.

Assessing generic strategies: an empirical investigation of two competing typologies in discrete manufacturing industries.
Strategic Management

Journal, v. 16, p. 75-83, 1995.

MILES, R. E. et al.

Organizational strategy, structure and process. Academy of Management Review, [s.n.], p. 546-562, July 1978.

MINTZBERG, H.

Generic strategies: toward a comprehensive framework. In: Advances in strategic management. Greenwich: JAI Press, 1988. v. 5. p. 1-67.

MINTZBERG, H.;

QUINN, J. B.

The strategy process: concepts, contexts, cases. 3. ed. Englewood Cliffs, NJ: PrenticeHall, 1996.

PMB.

Pharmaceutical Market Brazil. Institute of Marketing Statistics, Switzerland, 1999.

PORTER, M. E.

Competitive strategy: techniques for analysing industries and competitors. New York: The Free Press, 1980.

Competitive advantage: creating and sustaining competitive performance. New York: The Free Press, 1985. 
SILVA, J. F.

Desempenho de setores industriais: impacto das estratégias competitivas e colaborativas. Rio de Janeiro, 1997. Doutorado (Tese) - Departamento de Engenharia Industrial, Pontifícia Universidade Católica do Rio de Janeiro. 